\title{
Barbara Bleisch, Andrea Büchler (2020) Kinder wollen. Über Autonomie und Verantwortung
}

\author{
Carl Hanser Verlag, München, 303 Seiten, 22,00€, ISBN \\ 978-3-446-26575-2
}

\author{
Katharina Beier
}

Angenommen: 20. August 2021 / Online publiziert: 15. September 2021

(C) Der/die Autor(en) 2021

Die Erfüllung von Kinderwünschen mit Hilfe der Reproduktionsmedizin oder Dritter wirft vielfältige ethische, rechtliche und soziale Fragen auf. Mit ihrem Buch „Kinder wollen" haben die seit vielen Jahren zu diesem Themenkomplex publizierende Philosophin Barbara Bleisch und die Rechtswissenschaftlerin Andrea Büchler eine differenzierte, den Diskurs um moderne reprogenetische Verfahren sortierende Analyse vorgelegt. Im Fokus stehen fünf Dimensionen des ,Kinderwollens“ und - so muss man den Titel ergänzen - Elternwerdens: der Wunsch nach einem Kind, zu einem bestimmten Zeitpunkt, nach keinem Kind, einem bestimmten Kind sowie nach einem Kind mit Hilfe Dritter. Dabei treten die Autorinnen nicht nur als distanzierte Chronistinnen des ,breit gefächerten, vielstimmigen Gespräch[s]“ (S. 14) über das Kinderwollen hervor, sondern fügen diesem ihre eigene, philosophisch-ethische und rechtliche Expertise verbindende Stimme hinzu.

Bleisch und Büchler verorten die mit dem Kinderwollen verbundenen existenziellen Fragen im normativen Spannungsfeld von reproduktiver Freiheit und Verantwortung. Dabei treten sie für ein Verständnis reproduktiver Autonomie ein, das, über die Abwehr staatlicher Eingriffe hinaus, ein Recht auf Inanspruchnahme fortpflanzungsmedizinischer Assistenz umfasst. Im Rahmen eines Ansatzes, der seinen Ausgangspunkt ,,in der mit der entstehenden Beziehung einhergehenden Verantwortung“ (S. 55) hat, beanspruchen sie, die Bedeutung und Reichweite reproduktiver Autonomie als unverzichtbares, im Angesicht neuer reproduktionsmedizinischer Optionen jedoch „diffus gewordenes normatives Prinzip“ (S. 56f.) zu klären. Dabei räumen sie ein, dass es vernünftige Meinungsunterschiede gibt, ,die sich nicht dadurch auflösen lassen, dass man der anderen Seite Denkfehler nachweist“" (S. 20), schließen

\footnotetext{
Dr. Katharina Beier $(\bowtie)$

Institut für Ethik und Geschichte der Medizin, Universitätsmedizin Göttingen,

Humboldtallee 36, 37073 Göttingen, Deutschland

E-Mail: kbeier@gwdg.de
} 
aber zur Beschränkung reproduktiver Autonomie Argumente aus, die, wie z. B. das der Natürlichkeit oder des Kindeswohls, auf fragwürdigen Prämissen beruhen.

Auch wenn Kinderwünsche nicht ,grundlos“ (S. 95) sind, entziehen sie sich als „Herzensanliegen“ einer „Begründung, Analyse oder argumentativen Durchdringung“ (S. 73). Die Forderung, Kinder ,um ihrer selbst willen“ zu wollen, weisen Bleisch und Büchler daher ebenso als Karikatur von Elternschaft zurück, wie den Vorwurf, dass die Reproduktionsmedizin Kinder zu einem planbaren Lebensprojekt mache. Sich auf das schicksalhafte, unplanbare Leben mit Kindern einzulassen und die damit verbundene Verantwortung zu übernehmen, bleibe ein „Abenteuer“ (S. 76).

Luzide arbeiten Bleisch und Büchler die ambivalenten Implikationen reproduktiver Autonomie für verschiedene Anwendungskontexte heraus. Ob diese Autonomie fördern oder neue Zwänge erzeugen, hänge maßgeblich von den Rahmenbedingungen ab. So sehen die Autorinnen z. B. Leihmutterschaft als moralisch zulässig an, wenn diese auf einer persönlichen, auf Wertschätzung und Vertrauen basierenden Beziehung basiert. Auch seien Frauen in der Lage, sich selbstbestimmt für Social Freezing zu entscheiden, sofern es sich dabei um ein genuines Angebot handle. Einwände gegen späte Mutterschaft entlarven sie hingegen als Ressentiments. Da sich die Frage verantwortlicher Elternschaft für alle potenziellen Mütter und Väter stellt und das Kindeswohl keineswegs nur von deren Lebensalter abhängt, dürfe Elternschaft nicht an Bedingungen, wie z. B. Altersgrenzen, geknüpft werden. Ebenso widerspreche es der reproduktiven Autonomie, Eltern zu lizenzieren oder unter Berufung auf gesellschaftliche (z.B. klimapolitische) Erwägungen zu verlangen, gänzlich auf Kinder zu verzichten.

Für den Umgang mit pränatalen Tests argumentieren Bleisch und Büchler, dass Fragen der Zumutbarkeit und Lebensqualität nur von den Betroffenen selbst beantwortet werden könnten. Auch wenn das Angebot nicht-invasiver Pränataltests (NIPT) Menschen mit Behinderung nicht diskriminiere, sei die Sorge vor einer Routinisierung von NIPT sowie Perpetuierung negativer Werturteile ernstzunehmen. Um den Kern reproduktiver Autonomie nicht zu verraten, müsse dieser jedoch mit inkludierenden Maßnahmen, statt Einschränkungen persönlicher Entscheidungsfreiheit begegnet werden.

Wird der Wunsch nach einem bestimmten Kind häufig mit der Zeugung von „Designer-Babies“ gleichgesetzt, halten Bleisch und Büchler dem entgegen, dass die vorgeburtliche Selektion von Embryonen für einige Paare die einzige Möglichkeit ist, um ein eigenes Kind zu bekommen. Die Frage sei somit nicht, „ob die Präimplantationsdiagnostik überhaupt, sondern (..) zu welchen Zwecken sie zulässig sein soll“ (S. 182). Dies gelte erst recht für Keimbahneingriffe. Stellt die Prävention schwerer Krankheiten ein moralisch legitimes Ziel dar, verlaufe die Grenze für den Wunsch nach einem bestimmten Kind dort, wo ökonomische, gesundheitsoder bevölkerungspolitische Erwägungen drohen, in eine Pflicht zur Selektion oder Optimierung umzuschlagen.

Sind die neuen reprogenetischen Optionen Bleisch und Büchler zufolge ,weder Verdikt noch Verheißung“ (S. 268), haben sie doch einen Preis, insofern wir uns als Einzelne, aber auch als Gesellschaft unweigerlich zu diesen verhalten und Verantwortung für unser Tun wie auch Unterlassen übernehmen müssen. Ihre sorgfältig 
abwägende, abschließende Antworten vermeidende Analyse bietet dafür wertvolle Orientierung.

Funding Open Access funding enabled and organized by Projekt DEAL.

Open Access Dieser Artikel wird unter der Creative Commons Namensnennung 4.0 International Lizenz veröffentlicht, welche die Nutzung, Vervielfältigung, Bearbeitung, Verbreitung und Wiedergabe in jeglichem Medium und Format erlaubt, sofern Sie den/die ursprünglichen Autor(en) und die Quelle ordnungsgemäß nennen, einen Link zur Creative Commons Lizenz beifügen und angeben, ob Änderungen vorgenommen wurden.

Die in diesem Artikel enthaltenen Bilder und sonstiges Drittmaterial unterliegen ebenfalls der genannten Creative Commons Lizenz, sofern sich aus der Abbildungslegende nichts anderes ergibt. Sofern das betreffende Material nicht unter der genannten Creative Commons Lizenz steht und die betreffende Handlung nicht nach gesetzlichen Vorschriften erlaubt ist, ist für die oben aufgeführten Weiterverwendungen des Materials die Einwilligung des jeweiligen Rechteinhabers einzuholen.

Weitere Details zur Lizenz entnehmen Sie bitte der Lizenzinformation auf http://creativecommons.org/ licenses/by/4.0/deed.de.

Interessenkonflikt K. Beier gibt an, dass kein Interessenkonflikt besteht. 